\title{
Determination of Length and Width of a Line-Segment by Using a Hough Transform
}

\author{
Zezhong $\mathrm{Xu}^{1,2}$, Bok-Suk Shin ${ }^{1}$, and Reinhard Klette ${ }^{1}$ \\ 1 Department of Computer Science, The University of Auckland, \\ Auckland, New Zealand \\ 2 College of Computer Information Engineering, Changzhou Institute of Technology, \\ Changzhou, Jiangsu, China \\ zezhongx@gmail.com, \{b.shin,r.klette\}@auckland.ac.nz
}

\begin{abstract}
The standard Hough transform does not provide length and width of a line-segment detected in an image; it just detects the normal parameters of the line. We present a novel method for determining also length and width of a line segment by using the Hough transform. Our method uses statistical analysis of voting cells around a peak in the Hough space. In image space, the voting cells and voting values are analysed. The functional relationship between voting variance and voting angle is deduced. We approximate this relationship by a quadratic polynomial curve. In Hough space, the statistical variances of columns around a peak are computed and used to fit a quadratic polynomial function. The length and width of a line segment are determined simultaneously by resolving the equations generated by comparing the corresponding coefficients of two functions. We tested and verified the proposed method on simulated and real-world images. Obtained experimental results demonstrate the accuracy of our novel method for determining length and width of detected line segments.
\end{abstract}

Keywords: Hough transform, length, width, curve fitting.

\section{Introduction}

Line segments are important when analyzing geometric shapes in images for machine vision applications; see, for example, [16. In particular this problem also involves a need to extract parameters of line segments in images, such as width and length.

A class of methods for line detection applies least-square fitting; see, for example, 151719 22. These methods are in general sensitive to outliers; they require that feature points are clustered.

The Hough transform (HT) [18112324] defines an alternative class of methods. The basic HT does not provide length or width of a detected line segment; it only provides the two normal parameters $d$ and $\alpha$ of a line; see Eq. (1) below for those two parameters. This paper contributes to the HT subject.

In principle, the HT is able to detect the length of a line segment. After having the direction of a set of approximately collinear pixels detected, we can project 
the estimated collinear image features on the $x$ - or $y$-axis in image space; see, for example, [5 18 25]; the length of the line-segment is then determined as the Euclidean distance between the estimated two endpoints.

There are also HT methods which use the butterfly distribution in the Hough space, as identified in [10]. These butterfly-techniques have origins in methods proposed earlier. Akhtar 2] calculates the length of a detected line segment based on the spreading of voting cells in a column around the peak. Ioannou [12] estimates the line-segment length by analyzing the total vote values of cells in the peak column. In [3 4 13 14, the endpoints are detected by resolving simultaneously equations obtained by the first and the last non-zero-value voting cells in any two columns around the peak; the length is then again calculated as the Euclidean distance between the estimated two endpoints.

These methods detect the length besides the standard HT output of normal parameters of a detected line segment. But, they do not contribute to the calculation of the width of the line segment.

Du et al. 67] consider the complete parameter description of a line segment, defined by direction, length, width, and position. Here, length is obtained by measuring the vertical width of a butterfly wing. The width of a line segment is computed by comparing the actual voting value and theoretical voting values in a specific column. Reliable length and width are obtained using a Mean Square Error (MSE) estimation by considering multiple columns. This method is affected by image noise. The detection accuracy relies on a very fine quantization of the Hough space.

This paper proposes an HT method for obtaining the length and width of a detected line segment. The voting variance is analyzed in image space, and a $2^{\text {nd }}$ order functional relationship is deduced. In Hough space, the statistical variances of columns around a peak are computed and used to fit a quadratic polynomial function. Length and width of a line segment are determined by resolving the equations generated by comparing the corresponding coefficients of two functions.

The rest of the paper is organized as follows. Section 2 analysis the voting variance in image space. Section 3 introduces the voting distribution in Hough space, and calculates the length and width of a line segment. Section 4 provides experimental results. Section 5 concludes.

\section{Voting Analysis in Image Space}

Following [8], the standard Hough transform applies the following equation

$$
d=x \cdot \cos \alpha+y \cdot \sin \alpha
$$

for representing a straight line by normal parameters $\alpha$ and $d$. This representation was introduced in [20] when defining a transformation in continuous space, today known as the Radon transform; this transform is a generalization of the Hough transform. 
All pixels on the line-segment in an image vote for all possible cells $\left(\alpha_{i}, d_{j}\right)$ in Hough space. For a pixel, given a voting angle $\alpha_{i} \in[0, \pi)$, the corresponding $d_{j}$-value is computed. The cell $\left(\alpha_{i}, d_{j}\right)$ is voted for by increasing the voting value at this cell by 1 . Let $H_{i j}$ be the voting value of cell $\left(\alpha_{i}, d_{j}\right)$ in Hough space.

For a voting angle $\alpha_{i}$, the number of voting cells and voting values of each cell are analyzed first; then we deduce a functional relationship between voting variance and voting angle.

The actual normal parameters of a line segment are denoted by $\left(\alpha_{0}, d_{0}\right)$. Let $L$ and $T$ denote the length and the width of the line segment. For abbreviation, let $S$ and $C$ be short for the values of sine and cosine of $\left|\alpha_{i}-\alpha_{0}\right|$, respectively:

$$
S=\sin \left|\alpha_{i}-\alpha_{0}\right| \quad \text { and } \quad C=\cos \left|\alpha_{i}-\alpha_{0}\right|
$$

\subsection{Voting Cells and Voting Values}

Regarding a voting angle $\alpha_{i}$, the number of voting cells is proportional to the number of parallel bars intersected by the considered line-segment. The voting value $H_{i j}$, corresponding to the voting angle $\alpha_{i}$ and the distance $d_{j}$, is proportional to the length of the bar intersected by the line-segment.

For detecting line segments with different length and width, we consider two cases for estimating the number of voting cells and voting values.

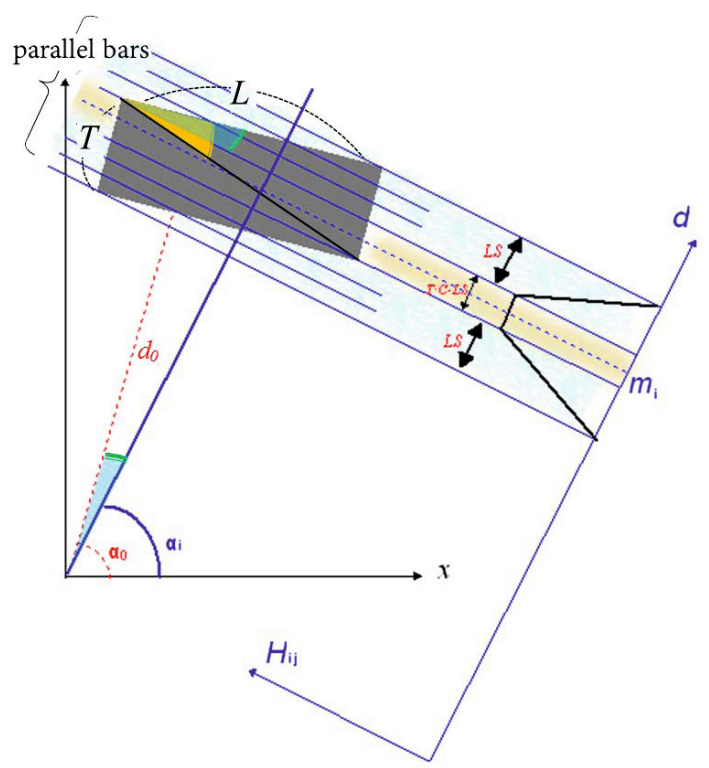

Fig. 1. The number of voting cells and voting values for $\left|\alpha_{i}-\alpha_{0}\right|<\arctan (T / L)$. Actual parameters are normal parameters $d_{0}$ and $\alpha_{0}$, and width $T$ and length $L$. For the remaining parameters in the figure see the text for explanations. 
For a voting angle $\alpha_{i}$, if $\left|\alpha_{i}-\alpha_{0}\right|<\arctan (T / L)$ then there are $T \cdot C+L \cdot S$ parallel bars crossing the considered line-segment in total. This is illustrated in Fig. 1. At the middle of the parallel bars, the number of voting cells equals

$$
T \cdot C-L \cdot S
$$

and the voting values are identical. On both outer sides of parallel bars, there are $L \cdot S$ voting cells for each side; and the voting values decrease to 0 gradually.

For a voting angle $\alpha_{i}$, if $\left|\alpha_{i}-\alpha_{0}\right|>\arctan (T / L)$ then there are $L \cdot S+T \cdot C$ parallel bars crossing the considered line-segment in total. This is illustrated in Fig. 2. At the middle of the parallel bars, the number of voting cells equals

$$
L \cdot S-T \cdot C
$$

and the voting values are identical. On both outer sides of the parallel bars, there are $T \cdot C$ voting cells on each side; and the voting values decrease to 0 gradually.

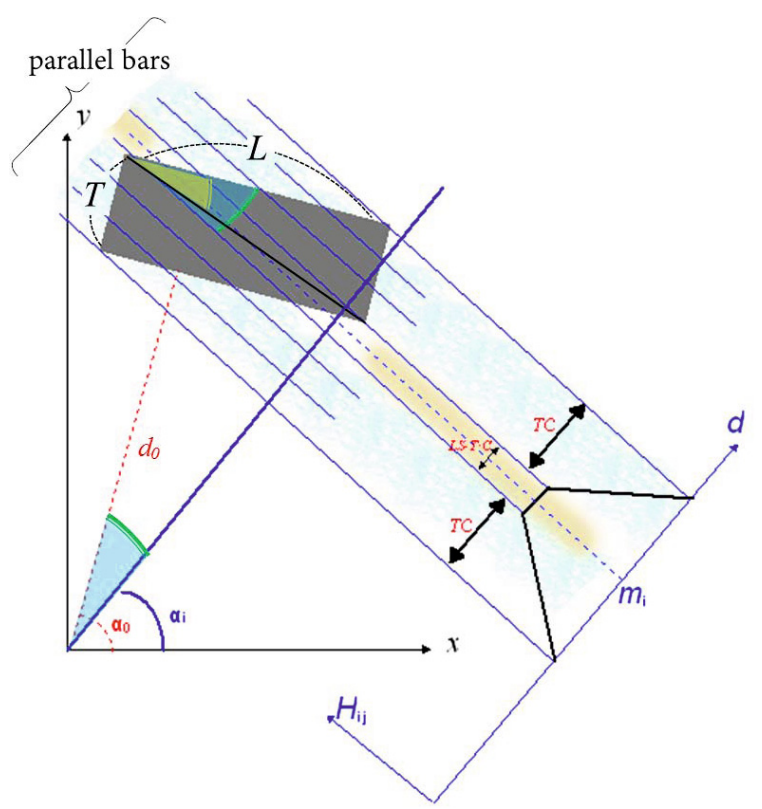

Fig. 2. The number of voting cells and voting values for $\left|\alpha_{i}-\alpha_{0}\right|>\arctan (T / L)$. See the text for explanations.

\subsection{Voting Variances}

In both cases for a voting angle $\alpha_{i}$, we consider the voting cells along the axis $d$ to be a random variable. The voting values of corresponding cells define a 
probabilistic density function. The voting variance $\sigma_{i}^{2}$, which corresponds to voting angle $\alpha_{i}$, is calculated based on the corresponding probabilistic density function.

For both discussed cases, the voting variance $\sigma_{i}^{2}$ is calculated as follows:

$$
\begin{aligned}
\sigma_{i}^{2} & =\frac{L^{2} \sin ^{2}\left|\alpha_{i}-\alpha_{0}\right|+T^{2} \cos ^{2}\left|\alpha_{i}-\alpha_{0}\right|}{12} \\
& =\frac{\left(L^{2}-T^{2}\right) \sin ^{2}\left|\alpha_{i}-\alpha_{0}\right|+T^{2}}{12}
\end{aligned}
$$

We only consider those voting cells around the peak in Hough space. It means that $\left|\alpha_{i}-\alpha_{0}\right|$ is small, and that we can approximate $\sin \left|\alpha_{i}-\alpha_{0}\right|$ by $\left|\alpha_{i}-\alpha_{0}\right|$. Thus we have the following:

$$
\begin{aligned}
\sigma_{i}^{2} & \approx \frac{\left(L^{2}-T^{2}\right)\left(\alpha_{i}-\alpha_{0}\right)^{2}+T^{2}}{12} \\
& =\frac{\left(L^{2}-T^{2}\right)\left(\alpha_{i}^{2}+\alpha_{0}^{2}-2 \alpha_{i} \alpha_{0}\right)+T^{2}}{12} \\
& =\frac{\left(L^{2}-T^{2}\right) \alpha_{i}^{2}-2 \alpha_{0}\left(L^{2}-T^{2}\right) \alpha_{i}+\left(L^{2}-T^{2}\right) \alpha_{0}^{2}+T^{2}}{12}
\end{aligned}
$$

This shows that the functional relationship between voting variance $\sigma^{2}$ and voting angle $\alpha$ can be approximated by a $2^{\text {nd }}$ order curve (called $f$ for later reference) as expressed in Eq. ([6).

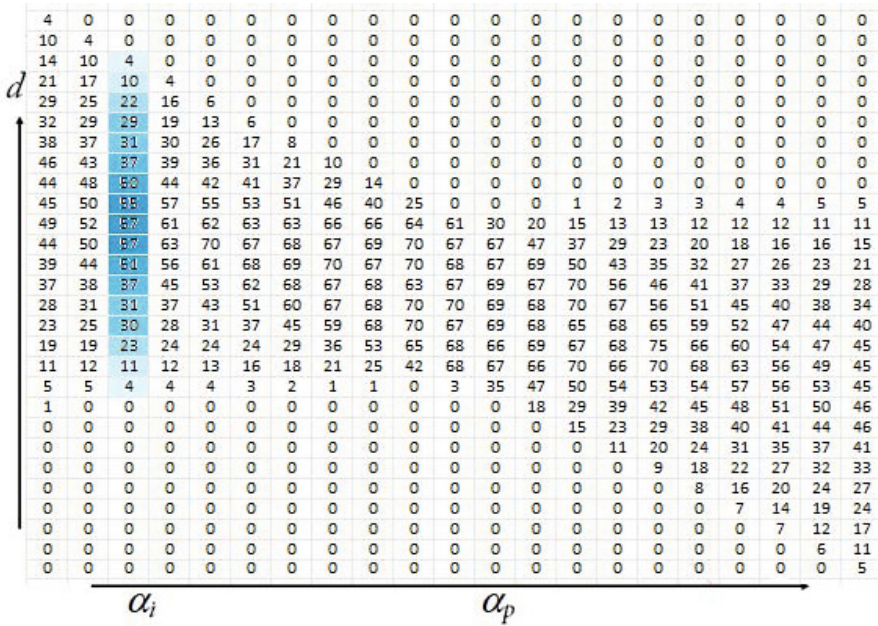

Fig. 3. Voting distribution in an $\alpha_{i}$ column for one "thick" line segment. Blue gradient cells illustrate that the voting values decrease gradually on both sides. 


\section{Statistical Distribution in Hough Space}

For a line segment in an image, all collinear pixels vote for all possible cells in the Hough space. Due to various uncertainties, the voting in a column is considered as being a random variable. The voting value at each cell defines a probabilistic distribution. We compute the statistical variances in columns near the peak and use them to fit a quadratic polynomial curve, called $g$ for later reference.

After voting, a peak is detected and represented by $\left(\alpha_{p}, d_{p}\right)$. This is just a coarse estimate for the actual normal parameters $\left(\alpha_{0}, d_{0}\right)$.

In that $\alpha_{i}$-column which is close to the peak $\alpha_{p}$, the middle cells have approximately identical voting values. Those voting values are larger than the voting values at outer cells. See Fig. 3 for an illustration.

\subsection{Statistical Variances}

For each column $\alpha_{i}$ in a peak region, the statistical mean $m_{i}$ and statistical variance $\sigma_{i}^{2}$ are computed as follows:

$$
\begin{aligned}
m_{i} & =\sum_{j \in W}\left[H_{i j} \cdot d_{j}\right] / \sum_{j \in W} H_{i j} \\
\sigma_{i}^{2} & =\sum_{j \in W}\left[H_{i j} \cdot\left(d_{j}-m_{i}\right)^{2}\right] / \sum_{j \in W} H_{i j}
\end{aligned}
$$

where $W$ defines the peak region in the Hough space.

\subsection{Quadratic Polynomial Curve Fitting}

Based on a voting analysis as discussed above, the functional relationship between statistical variance $\sigma^{2}$ and angle $\alpha$ can be approximated by a quadratic polynomial curve.

We fit a quadratic polynomial curve $g$ to pairs $\left(\sigma_{i}^{2}, \alpha_{i}\right)$, all calculated in the peak region. Formally, this is denoted by

$$
\begin{aligned}
g: \sigma^{2} & =g(\alpha) \\
& \triangleq e_{2} \alpha^{2}+e_{1} \alpha+e_{0}
\end{aligned}
$$

\subsection{Length and Width of Line-Segment}

We compute length and width of a detected line segment based on the coefficients of the fitted function.

Following Eqs. (6) and (8), we obtain the following equational system:

$$
\begin{aligned}
\left(L^{2}-T^{2}\right) / 12 & =e_{2} \\
-2 \alpha_{0}\left(L^{2}-T^{2}\right) / 12 & =e_{1} \\
\left(\left(L^{2}-T^{2}\right) \alpha_{0}^{2}+T^{2}\right) / 12 & =e_{0}
\end{aligned}
$$


By solving simultaneously those equations, the length $L$ and width $T$ of the line segment are as follows:

$$
\begin{aligned}
& L=\sqrt{12} \sqrt{e_{2}+e_{0}-\frac{e_{1}^{2}}{4 e_{2}}} \\
& T=\sqrt{12} \sqrt{e_{0}-\frac{e_{1}^{2}}{4 e_{2}}}
\end{aligned}
$$

This defines our novel closed-form solution.

\section{Experimental Results}

We tested and verified the proposed method for determining the length and width of a detected line segment. We used a set of simulated image data as well as real-world images.

Used simulated binary images are of size $200 \times 200$. Each image contains a representation of one digitized line-segment as well as background image noise. A background pixel is called noisy if it is black due to the generated background noise. For the digitised line segments we have all their parameters available, including length and width, defining the ground truth. The direction, position, length, and width of a synthesised line segment are generated randomly in our test data.

Figure 4 illustrates an example for line segment detection. The length and width are accurately calculated when applying the proposed method.

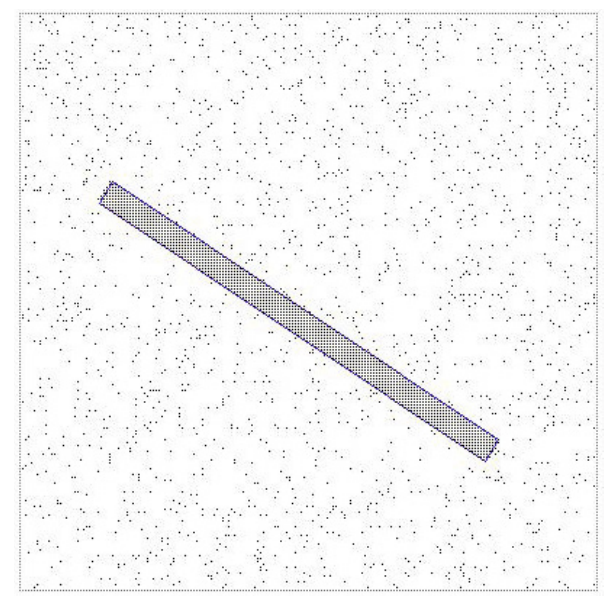

Fig. 4. Illustration of an example of our simulated binary images for determining the length and width for a line-segment with the proposed method. The blue box is drawn according to calculated length and width of the detected line-segment. 

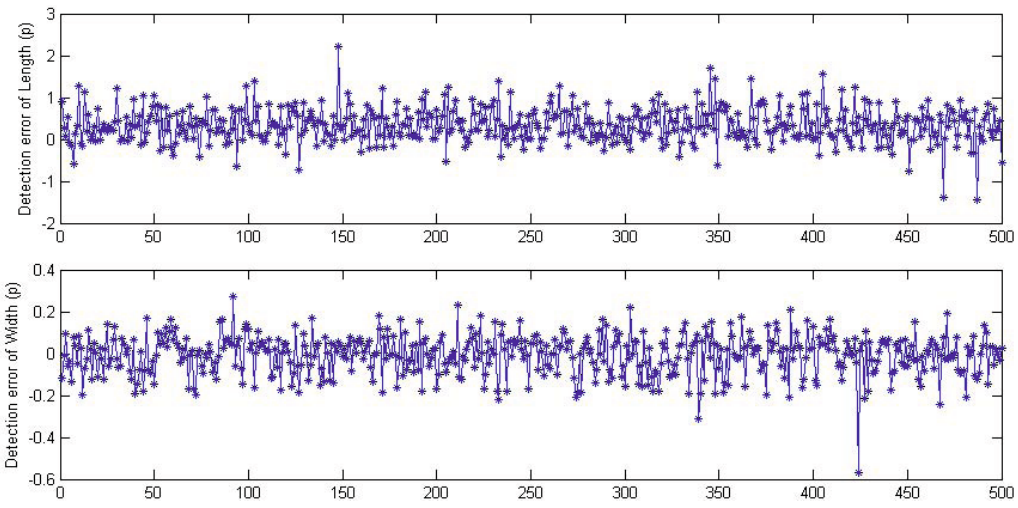

Fig. 5. Detection errors in the common case. Top: Length. Bottom: Width.
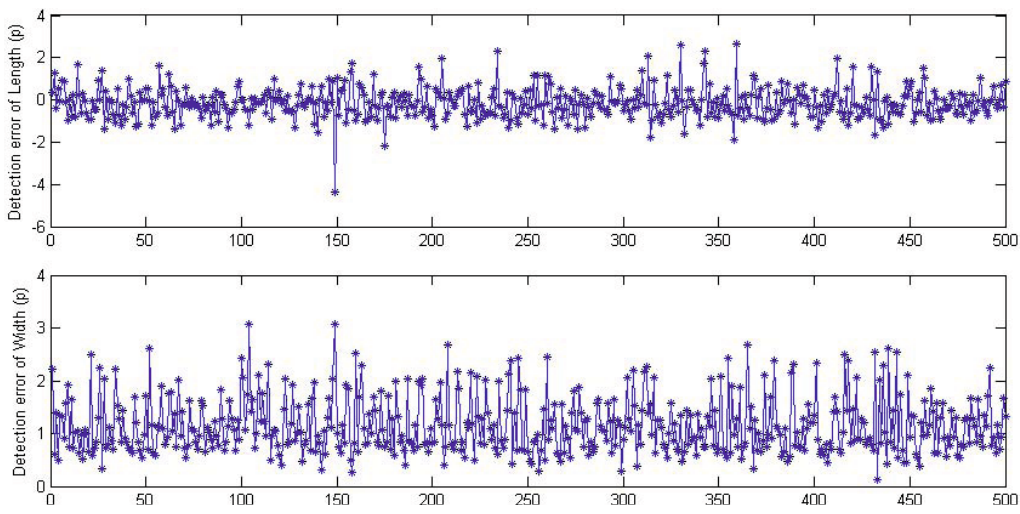

Fig. 6. Detection errors in the coarse-quantization case. Top: Length. Bottom: Width.
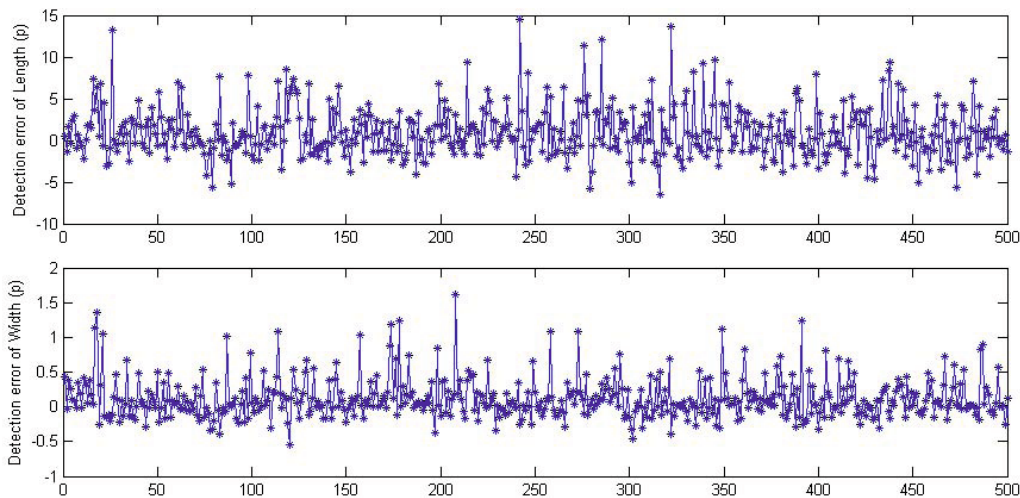

Fig. 7. Detection errors in the heavy-noise case. Top: Length. Bottom: Width. 
Our method focuses on the accuracy of length and width calculation for a single detected line segment. For the accuracy of length and width determination, three cases have been considered in terms of different quantization steps and noise scales.

In the common case there are no noisy pixels, and the quantization of the Hough space equals $(\Delta \alpha, \Delta d)=\left(1^{\circ}, 1 p\right)$ (the unit for $d$ is the pixel distance). For the coarse-quantization case, we set parameter quantization equal to $(\Delta \alpha, \Delta d)=$ $\left(4^{\circ}, 4 p\right)$. For the heavy-noise case, 1,000 noisy pixels are randomly generated in each of the $200 \times 200$ images used.

We generated 500 synthetic images randomly for each of the three cases and tested the proposed method. For each of the three cases, 500 resulting detection errors for length and width are documented by Figs. 5, 6, and 7.

In the common case, the calculated values for length and width are accurate. The mean errors of length and width are equal to 0.4853 and 0.0781 , respectively. When the Hough space is quantized at $(\Delta \alpha, \Delta d)=\left(4^{\circ}, 4 p\right)$, the mean errors of length and width are equal to 0.5796 and 1.1478 , respectively. The length detection is accurate, while the width detection is sensitive to the quantization interval $\Delta d$. By adding 1,000 noisy pixels, the mean errors of length and width are equal to 3.0772 and 0.2613 , respectively. The calculated width values are still accurate but length calculation is now effected by the given image noise.

For testing on recorded images, we use image sequences published in Set 5 of EISATS [9]. Images are of size $640 \times 480$. Those image sequences have been recorded for studying algorithms for vision-based driver assistance, in particular for algorithms detecting and tracking lane borders. (A review about visual lane analysis is given in [21]).
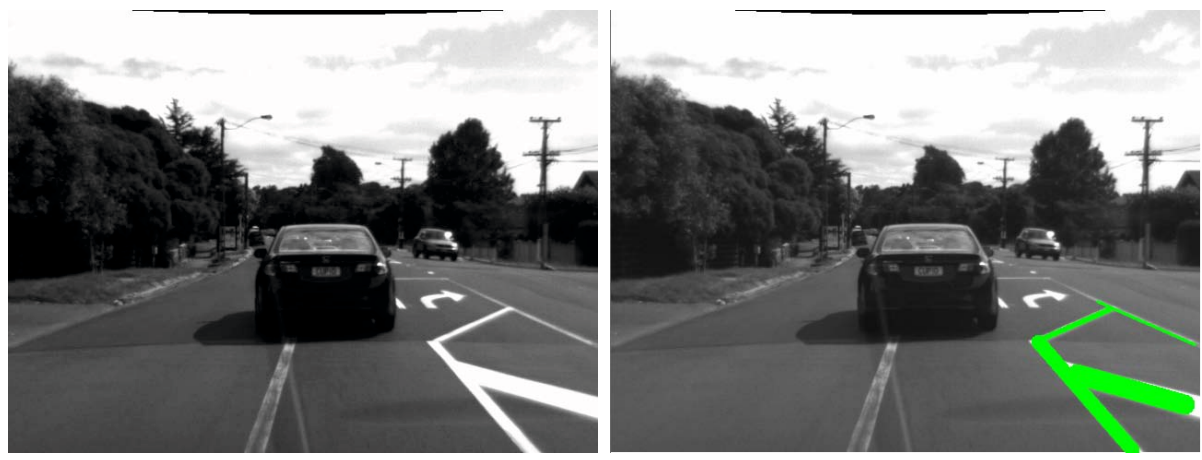

Fig. 8. Detection results for lane markers in real-world images. Left: Original images. Right: Detected lane markers.

Lane-detection results for one image of this data set is shown in Fig. 8. Only pixels in the lower half of the images are processed by supposing that lane borders are constrained to this image region. We are able to detect both frontiers (i.e. left and right border lines) of one lane marker as individual line segments 


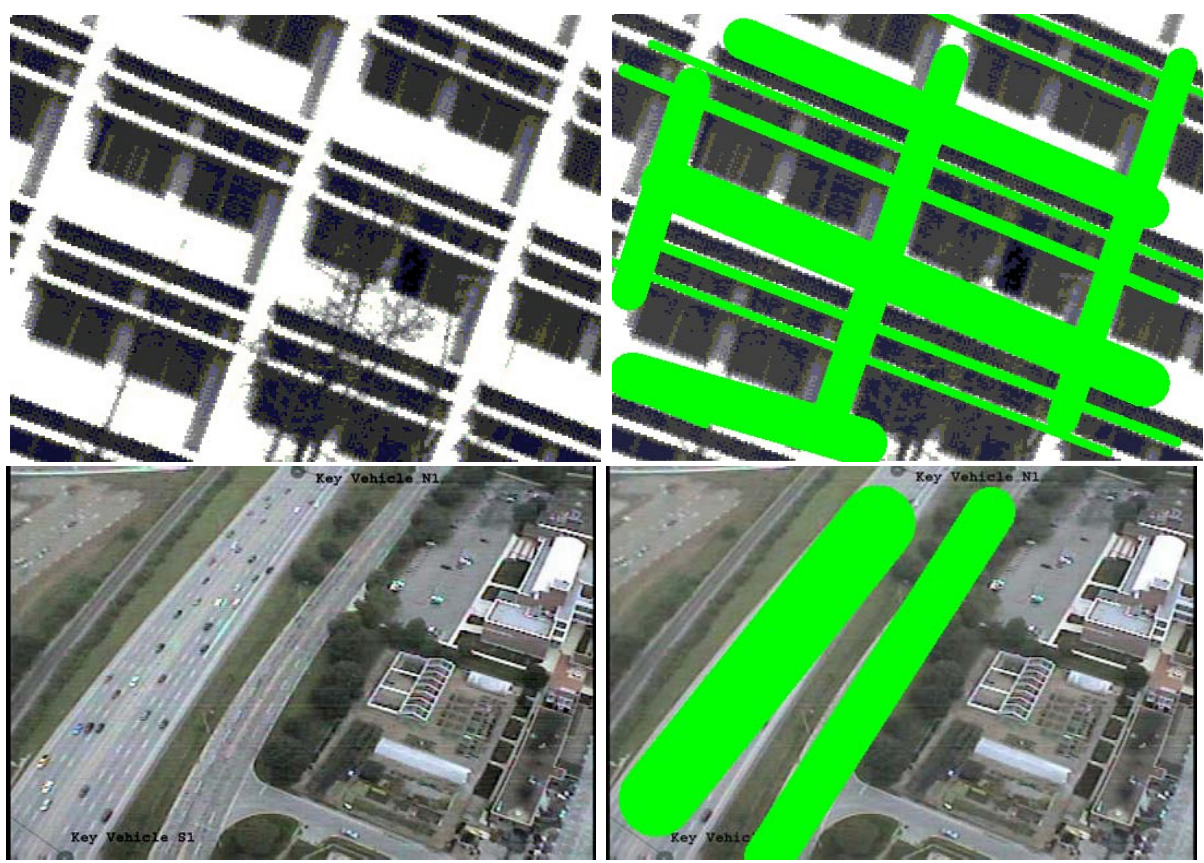

Fig. 9. Detection results for a building facade and road images. Left: Original images. Right: Detected result.

when using the accurate line detector reported in 23 . However, lane-border detection usually does not require such a fine and accurate line detection; it is more appropriate to detect one lane marker as a line segment of some width. This also supports the typically following step of lane-border tracking that only such line segments are accepted which do have a width within a given interval estimated for lane markers.

We also test the proposed method on building facade images and road images; see Fig. 9 for two examples, also showing detected lines. For the building facade image, all linear features with different length and width are detected. Two wide roads are detected in the shown aerial road view.

Line-segment features in images have varying lengths and widths. The proposed method calculates the length and width of these linear features using a Hough transform.

\section{Conclusions}

This paper proposes a novel method for line-segment length and width calculation using a Hough transform. We analyse the voting variance. We derive a functional relationship between the voting variance and the voting angle. This relation is approximated by a $2^{\text {nd }}$-order function $f$. Due to quantization errors and image noise, we consider voting in an $\alpha$-column as being a random variable, and 
voting values define a probabilistic distribution. We compute the corresponding statistical variances and use them to fit a quadratic polynomial curve $g$.

We obtain three equations by comparing the coefficients of functions $f$ and $g$. We calculate the length and width of a line segment by solving simultaneously these three equations. Various simulated and real-world images have been used for testing the proposed method, and also for illustrating new opportunities which are not yet available with previously specified line detection algorithms.

Experimental results verify the accuracy and feasibility of the proposed solution for line-segment length and width detection.

Acknowledgments. The first author thanks Jiangsu Overseas Research \& Training Program for University Prominent Young \& Middle-aged Teachers and Presidents for granting a scholarship to visit and undertake research at The University of Auckland.

\section{References}

1. Aggarwal, N., Karl, W.: Line detection in images through regularized Hough transform. IEEE Trans. Image Processing 15, 582-591 (2006)

2. Akhtar, M.W., Atiquzzaman, M.: Determination of line length using Hough transform. Electronics Letters 28, 94-96 (1992)

3. Atiquzzaman, M., Akhtar, M.W.: Complete line segment description using the Hough transform. Image and Vision Computing 12, 267-273 (1994)

4. Atiquzzaman, M., Akhtar, M.W.: A robust Hough transform technique for complete line segment description. Real-Time Imaging 1, 419-426 (1995)

5. Costa, L.F., Ben-Tzvi, B., Sandler, M.: Performance improvements to the Hough transform. In: UK IT 1990 Conference, pp. 98-103 (1990)

6. Du, S., Tu, C., van Wyk, B.J., Chen, Z.: Collinear segment detection using HT neighborhoods. IEEE Trans. Image Processing 20, 3912-3920 (2011)

7. Du, S., Tu, C., van Wyk, B.J., Ochola, E.O., Chen, Z.: Measuring straight line segments using HT butterflies. PLoS ONE 7(3), e33790 (2012)

8. Duda, R.O., Hart, P.E.: Use of the Hough transformation to detect lines and curves in pictures. Comm. ACM 15, 11-15 (1972)

9. EISATS: .enpeda.. image sequence analysis test site (2013), http://www.mi.auckland.ac.nz/EISATS

10. Furukawa, Y., Shinagawa, Y.: Accurate and robust line segment extraction by analyzing distribution around peaks in Hough space. Computer Vision Image Understanding 92, 1-25 (2003)

11. Hough, P.V.C.: Methods and means for recognizing complex patterns. U.S. Patent 3.069.654 (1962)

12. Ioannou, D.: Using the Hough transform for determining the length of a digital straight line segment. Electronics Letters 31, 782-784 (1995)

13. Kamat, V., Ganesan, S.: A robust Hough transform technique for description of multiple line segments in an image. In: Int. Conf. Image Processing, pp. 216-220 (1998)

14. Kamat, V., Ganesan, S.: Complete description of multiple line segments using the Hough transform. Image Vision Computing 16, 597-613 (1998) 
15. Kiryati, N., Bruckstein, A.M.: What's in a Set of Points? IEEE Trans. Pattern Analysis Machine Intelligence 14, 496-500 (1992)

16. Klette, R.: Concise Computer Vision. Springer, London (2014)

17. Netanyahu, N.S., Weiss, I.: Analytic line fitting in the presence of uniform random noise. Pattern Recognition 34, 703-710 (2001)

18. Nguyen, T.T., Pham, X.D., Jeon, J.: An improvement of the standard Hough transform to detect line segments. In: IEEE Int. Conf. Industrial Technology, pp. $1-6(2008)$

19. Qjidaa, H., Radouane, L.: Robust line fitting in a noisy image by the method of moments. IEEE Trans. Pattern Analysis Machine Intelligence 21, 1216-1223 (1999)

20. Radon, J.: Über die Bestimmung von Funktionen durch ihre Integralwerte längs gewisser Mannigfaltigkeiten. Berichte Sächsische Akademie Wissenschaften, Math.Phys. Kl. 69, 262-267 (1917)

21. Shin, B.-S., Xu, Z., Klette, R.: Visual lane analysis and higher-order tasks: A concise review. Machine Vision Applications (to appear, 2014)

22. Weiss, I.: Line fitting in a noisy image. IEEE Trans. Pattern Analysis Machine Intelligence 11, 325-329 (1989)

23. Xu, Z., Shin, B.-S.: Line segment detection with Hough transform based on minimum entropy. In: Klette, R., Rivera, M., Satoh, S. (eds.) PSIVT 2013. LNCS, vol. 8333, pp. 254-264. Springer, Heidelberg (2014)

24. Xu, Z., Shin, B.-S.: A statistical method for peak localization in Hough space by analysing butterflies. In: Klette, R., Rivera, M., Satoh, S. (eds.) PSIVT 2013. LNCS, vol. 8333, pp. 111-123. Springer, Heidelberg (2014)

25. Yamato, J., Ishii, I., Makino, H.: Highly accurate segment detection using Hough transformation. Systems and Computers in Japan 21, 68-77 (1990) 